STABILITY IN PROBABILITY

BANACH CENTER PUBLICATIONS, VOLUME 90

INSTITUTE OF MATHEMATICS

POLISH ACADEMY OF SCIENCES

WARSZAWA 2010

\title{
OPTIMAL STOPPING OF A 2-VECTOR RISK PROCESS
}

\author{
KRZYSZTOF SZAJOWSKI \\ Institute of Mathematics and Computer Science, Wrockaw University of Technology \\ Wybrzė̇e Wyspiańskiego 27, Wroctaw, Poland \\ and Institute of Mathematics, Polish Academy of Sciences \\ E-mail: Krzysztof.Szajowski@pwr.wroc.pl
}

\begin{abstract}
The following problem in risk theory is considered. An insurance company, endowed with an initial capital $a>0$, receives insurance premiums and pays out successive claims from two kind of risks. The losses occur according to a marked point process. At any time the company may broaden or narrow down the offer, which entails the change of the parameters of the underlying risk process. These changes concern the rate of income, the intensity of the renewal process and the distribution of claims. Our goal is to find the best moment for changes which is the moment of maximal value of the capital assets. Based on the representation of stopping times for piecewise deterministic processes and the dynamic programming method the solution is derived for the finite and infinite horizon model.
\end{abstract}

\section{Introduction}

1.1. Preliminaries. Let us consider an insurance company having an initial capital $a>0$ which insures two kind of risks. The $i$-th risk makes the stream of insurance premiums with the constant rate $c_{i}$ and pays out successive claims, which are represented by i.i.d. random variables $X_{i, 1}, X_{i, 2}, \ldots$ with cumulative distribution function $H_{i}$. The losses related to the $i$-th risk occur according to the renewal process $\left\{N_{i}(t), t \geq 0\right\}$, where $N_{i}(t), i=1,2$, is the number of claims up to time $t$ in the stream of the risk $i$. The renewal processes are mutually independent and they are independent of the sequence of claims. The 2 -vector process $\left(N_{1}(t), N_{2}(t)\right), t \geq 0$ can be represented also by a sequence of random variables $T_{n}$ taking values in $[0, \infty]$ such that

$$
\begin{gathered}
T_{0}=0, \\
T_{n}<\infty \quad T_{n}<T_{n+1},
\end{gathered}
$$

2010 Mathematics Subject Classification: Primary 60G40, 60K99; Secondary 90A46.

Key words and phrases: risk reserve process, optimal stopping, dynamic programming, Nash equilibrium, Elfving problem, capital assets.

The paper is in final form and no version of it will be published elsewhere. 
for $n \in \mathbb{N}$, and a sequence of $\{1,2\}$-valued random variables $Z_{n}$ for $n \in \mathbb{N} \cup\{0\}$ (see Brémaud (1981) Ch. II, Jacobsen (2006)). The random variable $T_{n}$ denotes the moment of the $n$-th claim $\left(T_{0}=0\right)$ from any stream of risks and the random variable $Z_{n}$ indicates to which stream of risks the $n$-th claim belongs. The processes $N_{i}(t)$ can be defined by the sequence $\left\{\left(T_{n}, Z_{n}\right)\right\}_{n=0}^{\infty}$ as follows:

$$
N_{i}(t)=\sum_{n=1}^{\infty} \mathbb{I}_{\left\{T_{n} \leq t\right\}} \mathbb{I}_{\left\{Z_{n}=i\right\}} .
$$

Both the 2-variate process $\vec{N}(t)=\left(N_{1}(t), N_{2}(t)\right)$ and the double sequence $\left\{\left(T_{n}, Z_{n}\right)\right\}_{n=0}^{\infty}$ are called 2-variate point processes.

Let us define for $i \in\{1,2\}$ and $k \in \mathbb{N}$ the sequence

$$
\begin{aligned}
n_{i, 0} & =0, \\
n_{i, k+1} & =\inf \left\{n>n_{i, k}: Z_{n}=i\right\}
\end{aligned}
$$

and put $\sigma_{i, k}=T_{n_{i, k}}$. Let us define random variables $S_{i, n}=\sigma_{i, n}-\sigma_{i, n-1}$ and assume that they are i.i.d. with continuous, cumulative distribution function $F_{i}(t)=\mathbf{P}\left(S_{i, n} \leq t\right)$ and the conditional distribution function $F_{i}^{s}(t)=\mathbf{P}\left(S_{i, n} \leq t \mid S_{i, n} \geq s\right)$. Define the balance between premium and collection of payoffs covering the claims $R_{i}(t)=c_{i} t-\sum_{s=1}^{N_{i}(t)} X_{i, s}$ in the risk $i$.

The considered processes allow to describe the reserves of insurance companies. Management decisions concerning the portfolio of risks change parameters of the processes. The main idea is to find a good moment to manage the assets. Various approaches are taken into account. The optimal stopping problems for processes which are similar to risk process have been considered by Davis (1993), Boshuizen and Gouweleeuw (1993), Boshuizen (1994). These results are described in detail later. Here we would like to mention that each group of risks can be tackled separately. The interference consists in successive control of each risk. It leads to switching between processes and stopping them. Observing risk processes and suggestions the decision makers choose one of the considered processes, based on the knowledge from the past, the best moment is found for switching between prescribed processes. In such a way one can model the case when the insurance company insures a risk modeled by the first process and at some moment $s$ it changes this process to the second one by broadening the offer to appeal to a wider range of customers. The optimal choice of the intervention and switching time and next stopping of the final risk process leads to the double stopping problem for stochastic processes. Multiple optimal stopping problems have been treated by many authors. The early formulation for discrete time double indexed processes was given by Haggstrom (1967) and for the discrete time Markov processes has been considered by Eidukjavicjus (1979), Nikolaev (1979, 1998) and Stadje (1985).

If there are two decision makers and their aims are in contradiction, but each of them can choose one moment of modification or stopping, the game model could be an appropriate approach. The first paper on the competitive stopping of discrete time stochastic processes has been presented by Dynkin (1969). Many extensions, modifications and applications of the Dynkin game have been investigated. Only a few of them are strictly related to risk processes. 
1.2. Formal description of the model. Let $\left\{\left(T_{n}, Z_{n}\right)\right\}_{n=0}^{\infty}$ (a sequence of pairs) be a 2 -variate point process $(\{1,2\}$-marked point process $)$ defined on $(\Omega, \mathcal{F}, \mathbf{P})$. Using the notations of the previous section there are three point processes $\left\{T_{i, n}\right\}_{n=1}^{\infty}$, where $T_{0, n}=T_{n}$ by convention, and $T_{Z_{n}, N_{Z_{n}}\left(T_{n}\right)}=T_{n}$. There are also three renewal processes $\left\{\left(T_{i, n}, X_{i, n}\right)\right\}_{n=1}^{\infty}, i=0,1,2$, where $X_{0, n}=X_{n}$ by convention, and $X_{Z_{n}, N_{Z_{n}}\left(T_{n}\right)}=X_{n}$. The following $\sigma$-fields generated by the history of the $\{1,2\}$-marked renewal processes are defined

$$
\mathcal{F}_{t}^{\{1,2\}}=\sigma\left(X_{1}, T_{1}, Z_{1} \ldots, X_{N(t)}, T_{N(t)}, Z_{N(t)}\right),
$$

for $t \geq 0$. These $\sigma$-fields can be defined as $\mathcal{F}_{t}^{\{1,2\}}=\sigma\left\{N_{i}(s), 0 \leq s \leq t, i=1,2\right\}$.

Definition 1.1. Let $\mathcal{T}$ be a set of stopping times with respect to the $\sigma$-fields $\left\{\mathcal{F}_{t}\right\}, t \geq 0$, defined by (4). The restricted sets of stopping times

$$
\mathcal{T}_{n, K}=\left\{\tau \in \mathcal{T}: \tau \geq 0, T_{n} \leq \tau \leq T_{K}\right\}
$$

for $n \in \mathbb{N}, n<K$ are subsets of $\mathcal{T}$. The elements of $\mathcal{T}_{n, K}$ are denoted $\tau_{n, K}$.

In the optimization problems formulated in the sequel, a crucial role is played by the representation of stopping times according to the following lemma (see Brémaud (1981) Appendix A.2, cf. also Davis (1993) Th. A2.3).

LEMMA 1.2. If $\tau \in \mathcal{T}_{n, K}$, then there exists a positive, $\mathcal{F}_{n}$-measurable, random variable $R_{n}$ such that

$$
\tau \wedge T_{n+1}=\left(T_{n}+R_{n}\right) \wedge T_{n+1}, \text { a.s. }
$$

It is convenient to diversify the form of $\tau$ based on information from the $Z_{n}$ sequence. We have for $i, j \in\{1,2\}, i \neq j$,

$$
\tau \wedge T_{j, N_{j}\left(T_{i, n}\right)+1} \wedge T_{i, n+1}=\left(T_{i, n}+R_{i, n}\right) \wedge T_{j, N_{j}\left(T_{i, n}\right)+1} \wedge T_{i, n+1}, \text { a.s. }
$$

where $R_{i, n}$ is $\mathcal{F}_{i, n}=\mathcal{F}_{T_{i, n}}$-measurable. This very precise description of the class of $\mathcal{F}_{t^{-}}$ stopping times is convenient in solution of various optimization problems for piecewise deterministic processes. Roughly speaking, $\tau \in \mathcal{T}$ stops a process at a moment of claims from the risk $i \in\{1,2\}$ or after this moment but not later than a new claim appears in any stream of risks. In the class of such strategies the optimization problem can be split into several different steps. There are decisions at the moment of claims and between the claims. This observation is justified by considerations of section 3 and 4 (see also Boshuizen and Gouweleeuw (1993)).

Let us consider the moment $\sigma_{i, k}=T_{n_{i, k}}$. It is the moment of a $k$-th event in the $i$-th stream of claims. The last event in the $j$-th stream of claims before $\sigma_{i, k}$ has appeared at $\sigma_{j, s_{k}^{\star}}=T_{j, n_{i, s_{k}^{\star}}}$, where $n_{j, s_{k}^{\star}}=\max \left\{n \leq n_{i, k}: Z_{n}=j\right\}=\max \left\{n_{j, s} \leq n_{i, k}: s \in \mathbb{N}\right\}$ and $s_{k}^{\star}=\arg \max \left\{n_{j, s} \leq n_{i, k}: s \in \mathbb{N}\right\}$. It follows that except $S_{i, n}$, the time between claim from the stream $i$, we have $S_{i, n}^{\star}=\sigma_{i, n}-\sigma_{j, s_{n}^{\star}}$, the time between claims in different stream of risks.

1.3. Risk process and economic environment. When a real enterprise is considered in an economic environment the effects of interest rate and inflation rate have to be taken into account. By interest rate we mean that the capital increases in time due to investments in money markets or risk-less bonds. The interest is modeled by $e^{\alpha(t)}$ as the 
value at time $t$ of the monetary unit invested at time 0 . The function $\alpha: \mathbb{R}^{+} \rightarrow \mathbb{R}^{+}$is increasing by assumption with the condition $\alpha(0)=0$. If $\alpha(t)$ is absolutely continuous, then $\alpha(t)=\int_{0}^{t} \delta(s) d s$, where the nonnegative function $\delta(t)$ is called the force of interest at time $t$ (or spot rate). The inflation means that a monetary unit at time 0 has the value $e^{-\beta(t)}$ at time $t$. The function $\beta(t)$ is a certain nonnegative, continuous function with the condition $\beta(0)=0$. The coefficient $e^{-\beta(t)}$ is called the discount factor. If $\beta(t)$ is absolutely continuous and $\beta(t)=\int_{0}^{t} \gamma(s) d s$ for some positive function $\gamma: \mathbb{R}^{+} \rightarrow \mathbb{R}^{+}$, then $\gamma(\cdot)$ is called the instantaneous inflation rate at time $t$. The discounted risk reserve process has been considered by many authors (see e.g. Rolski et al. (1998) Ch. 11.4). The optimal stopping problem for the risk process in the randomly varying economic environment has been considered by Schöttl (1998) and Muciek and Szajowski (2007). We assume in this paper that the effects of interest and inflation are canceling out.

1.4. Remarks on the terminology. Taking the most important features of a real problem into the mathematical description we usually get a universal model. It may happen that one and the same model can describe different situations which seemingly do not have too much in common. This results in different names for the same elements of the mathematical formulation. The considered processes are not an exception. This is broadly discussed in Rolski et al. (1998).

The general risk reserve model is defined as follows:

1. random epochs $T_{0}, T_{1}, T_{2}, \ldots$ with $T_{0}<T_{1}<T_{2}<\ldots$ at which the claims occur, where the random variables $T_{n}$ can be discrete or continuous;

2. the corresponding positive, individual or aggregate, claim size $X_{1}, X_{2}, \ldots$;

3. the initial risk reserve $a \geq 0$;

4. the premiums which are collected at a constant rate $c>0$, so that the premium income is a linear function of time.

The sequence $\left\{\left(T_{n}, X_{n}\right)\right\}$ of arrival epochs and claim sizes can be described equivalently by the inter-occurrence times $S_{n}=T_{n}-T_{n-1}$ and the claim sizes $X_{n}$. The sequence $\left\{T_{n}\right\}$ is called a point process and $\left\{\left(T_{n}, X_{n}\right)\right\}$ a marked point process (see Rolski et al. (1998) Ch. 12). We can define for further use the cumulative arrival process $U_{t}=\sum_{i=1}^{\infty} X_{i} \mathbb{I}_{\left\{T_{i} \leq t\right\}}=\sum_{i=1}^{N_{t}} X_{i} . U_{t}$ is the aggregate amount of all claims arriving in the interval $(0, t]$ and $N_{t}$ is the counting process $N_{t}=\sum_{i=1}^{\infty} \mathbb{I}_{\left\{T_{i} \leq t\right\}}$. The risk reserve process $\left\{R_{t}\right\}, t \geq 0$ is given by $R_{t}=a+c t-U_{t}$ and the claim surplus process $\left\{\xi_{t}\right\}, t \geq 0$ is defined as $\xi_{t}=U_{t}-c t$. The extension of the risk reserve model which is under consideration in the paper is introduced in Section 1.1 .

2. The risk processes and the optimization problems. Let us consider the above introduced risk processes as possible items used in the description of insurance companies. The following possibilities will be analyzed in the paper.

1. Both risks are parts of the capital assets $U(t), t \geq 0$, of the insurance company

$$
U(t)=a+\left(c_{1}+c_{2}\right) t-\sum_{s=1}^{N_{1}(t)} X_{1, s}-\sum_{s=1}^{N_{2}(t)} X_{2, s}
$$


Based on these two risk processes we can define different optimization problems. Let $g(u, t)=g_{1}(u) \mathbb{I}_{\{t \geq 0\}}$, where $g_{1}$ is a utility function. The return at time $t$ is $\{Z(t), t \geq 0\}$ and it is given by

$$
Z(t)=g\left(U_{t}, t_{0}-t\right) \prod_{j=0}^{N(t)} \mathbb{I}_{\left\{U_{T_{j}}>0\right\}}=g\left(U_{t}\right) \mathbb{I}_{\left\{U_{s}>0, s \leq t\right\}}
$$

The optimal stopping problem for the process $Z(t)$ is investigated.

$$
\begin{aligned}
V_{1}(a) & =\mathbf{E} g\left(U\left(\tau^{\star}\right)\right)=\sup _{\tau} \mathbf{E} g(U(\tau)), \\
V_{2}(a) & =\mathbf{E} g\left(a+R_{1}\left(\tau^{\star}\right)+R_{2}\left(\sigma^{\star}\right)\right)=\sup _{\tau, \sigma} \mathbf{E} g\left(a+R_{1}(\tau)+R_{2}(\sigma)\right), \\
V_{3}(a) & =\mathbf{E} g\left(a+\tilde{c}_{1}\left(\sigma^{\star}-\tau^{\star}\right)+R_{1}\left(\tau^{\star}\right)+R_{2}\left(\sigma^{\star}-\tau^{\star}\right)\right) \\
& =\sup _{\tau<\sigma} \mathbf{E} g\left(a+c_{1} \tau-\sum_{i=1}^{N_{1}(\tau)} X_{1, i}+\widetilde{c}_{1}(\sigma-\tau)+c_{2} \sigma-\sum_{i=1}^{N_{2}(\sigma-\tau)} X_{2, i}\right) .
\end{aligned}
$$

2. This paper focuses mainly on problem (12). The insurance company insures a risk modeled by the first process and in some moment $s$ it changes the risk process to the second one by broadening the offer to appeal to a wider range of customers. Let $U(s, t)$ be the value of the capital assets at time $t$, if the change of the parameters took place at time $s$, then

$$
U(s, t)= \begin{cases}a+c_{1} t-\sum_{n=0}^{N_{1}(t)} X_{n} & \text { if } t \leq s, \\ a+c_{1} s-\sum_{n=0}^{N_{1}(s)} X_{n}+c_{2}(t-s)-\sum_{n=0}^{N_{2}(t-s)} \tilde{X}_{n} & \text { if } t>s,\end{cases}
$$

where $X_{0}=0, \widetilde{X}_{0}=0$. It is convenient to introduce the classical Cramér-Lundberg risk process

$$
U_{1}(t)=a+c_{1} t-\sum_{n=0}^{N_{1}(t)} X_{n},
$$

which is the base of many models widely discussed in the literature (see Azcue and Muler (2005), Muciek (2002), Rolski et al. (1998)). Additionally, let

$$
U_{2}(t)=c_{2} t-\sum_{n=0}^{N_{2}(t)} \tilde{X}_{n}
$$

Then, the formula 13 reduces to

$$
U(s, t)= \begin{cases}U_{1}(t) & \text { if } t \leq s \\ U_{1}(s)+U_{2}(t-s) & \text { if } t>s\end{cases}
$$

3. The model of the risk process in the formula 13 allows to model the assets of more than one insurer. There are two companies which insure the risks modeled by the first and the second process, respectively. They agree to re-insure each other. This means that both companies share the risks in some proportion. The possible questions under consideration for such models are subject of another investigation.

The companies measure the performance of their business by utility functions $g$ defined on the state space of the process $U(t), t \geq 0$. Based on the history of the renewal 
processes we are looking for the best moment for changes of the policy. This leads to the optimization problem having the form given by 10 . The problem formulated in 12 ) has been tackled by Karpowicz and Szajowski (2007). The formulation at (11) should be discussed in the context of reinsurance or changes in the collection of risks. It is an open problem.

Assumption 1. The utility function $g$ is bounded, continuous, nondecreasing and differentiable.

2.1. The optimal stopping of the risk process with two types of claims. The random variables $S_{i, n}=\sigma_{i, n}-\sigma_{i, n-1}$ are times between successive claims of the $i$ type. Assume that they are i.i.d. with continuous, cumulative distribution function $F_{i}$, $i \in\{1,2\}$. The size of the demand $X_{i, n}$ has the distribution $H_{i}, i \in\{1,2\}$.

The set of stopping times with respect to $\left\{\mathcal{F}_{t}\right\}_{t \geq 0}$ is denoted by $\mathcal{T}$. There is a nice and useful representation of the stopping time given by (7) in Lemma 1.2 Let us denote $\mathcal{T}_{i, n, K}=\left\{\tau \in \mathcal{T}_{K}: T_{i, n} \leq \tau \leq T_{K}\right\}$. If $\tau \in \mathcal{T}_{i, n, K}$, then there exists a positive, $\mathcal{F}_{i, n^{-}}$ measurable, random variable $R_{i, n}$ such that

$$
\tau \wedge T_{j, N_{j}\left(T_{i, n}\right)+1} \wedge T_{i, n+1}=\left(T_{i, n}+R_{i, n}\right) \wedge T_{j, N_{j}\left(T_{i, n}\right)+1} \wedge T_{i, n+1}, \text { a.s. }
$$

The aim of the decision maker is to find the stopping time $\tau_{K}^{\star} \in \mathcal{T}_{K}$ such that

$$
\mathbf{E} \mathbb{G}\left(\tau_{K}^{\star}\right)=\sup _{\tau \in \mathcal{T}_{K}} \mathbf{E} \mathbb{G}(\tau),
$$

where

$$
\mathbb{G}(t)=g\left(U_{t}, t_{0}-t\right) \prod_{j=0}^{N(t)} \mathbb{I}_{\left\{U_{T_{j}>0}\right\}}
$$

and $U(t)$ is given by (8). In order to find the optimal stopping time $\tau_{K}^{\star}$ according to the definition (17), we first consider the optimal stopping times $\tau_{i, n, K}^{\star}$ such that

$$
\Gamma_{i, n, K}=\mathbf{E}\left(\mathbb{G}\left(\tau_{i, n, K}^{\star}\right) \mid \mathcal{F}_{i, n}\right)=\operatorname{ess}_{\tau \in \mathcal{T}_{i, n, K}} \mathbf{E}\left(\mathbb{G}(\tau) \mid \mathcal{F}_{i, n}\right),
$$

for $i \in\{1,2\}, n=0,1, \ldots, K$ and using backward induction on $i$ and $n$ as in the dynamic programming, we will obtain $\tau_{K}^{\star}=\tau_{0, K}^{\star}=\tau_{0,0, K}^{\star}$ (the claims at time 0 for both types of risks are 0 by assumption).

3. A fixed number of claims. In this section we find the form of an optimal stopping time in the finite horizon case, which means the optimal stopping time in the class $\mathcal{T}_{0, K}$, where $K$ is finite and fixed (the number of claims is fixed, but the time of the $K$ th claim, i.e. the time horizon, remains random). This is a technical assumption which allows to get a solution for a finite number of claims and next to extend it to an infinite number of claims. The structure of the risk process suggests to take into account, in the construction of the optimal stopping time, the information about the source of the successive claims and the time of its appearance.

First we present the dynamic programming solution satisfying

$$
\Gamma_{i, n, K}=\operatorname{ess} \sup \left\{\mathbf{E}\left(\mathbb{G}(\tau) \mid \mathcal{F}_{i, n}\right): \tau \in \mathcal{T}_{i, n, K}\right\},
$$


for $n=K, K-1, \ldots, 1$ and $i=1,2$. Then in Corollary 3.3 we find optimal stopping times $\tau_{n, K}^{*}, \tau_{K}^{*}$ and the optimal mean values of the return related to them.

Let $\mu_{0}=1$ and $\mu_{n}=\prod_{j=1}^{n} \mathbb{I}_{\left\{U_{T_{j}}>0\right\}}$. Then $\Gamma_{i, K, K}=\mathbb{G}\left(T_{i, K}\right)=g\left(U_{T_{i, K}}, t_{0}-T_{i, K}\right) \mu_{K}$. Note that the sum of claims from (8) can be expressed as

$$
\sum_{n=0}^{N_{1}(t)} X_{1, n}+\sum_{n=0}^{N_{2}(t)} X_{2, n}=a+\left(c_{1}+c_{2}\right) t-U_{t} .
$$

Let us define, for $\xi>0$ such that there is no jump between $t$ and $t+\xi$,

$$
d_{t, \xi, U_{t}}=U_{t+\xi}-U_{t}=\left(c_{1}+c_{2}\right) \xi
$$

then we have

$$
\mu_{i, K}=\mu_{i, K-1} \mathbb{I}_{\left\{U_{T_{i, K-1}}+d_{T_{i, K-1}, \zeta_{i, K}, U_{T_{i, K-1}}}-X_{i, K}>0\right\}}
$$

Similarly to Muciek (2002), Theorem 1, from 20 and 21) we get the dynamic programming equations for $\left\{\Gamma_{i, n, K}\right\}_{n=0}^{K}, i=1,2$.

THEOREM 3.1. For the given horizon $t_{0}$, the $\{1,2\}$-marked renewal processes and the fixed number of claims $K$, the optimal expected value of the stopping problem, with the restricted sets of stopping times $\mathcal{T}_{i, n, K}$, fulfills the recursive equations:

(i) For $n=K-1, K-2, \ldots, 0, i=1,2$,

$$
\begin{aligned}
\Gamma_{i, n, K}= & \operatorname{ess} \sup \left\{\mu_{n} \bar{F}_{i}(\xi) \bar{F}_{j}^{T_{i, n}}\left(\xi+T_{i, n}-T_{j, N_{j}\left(T_{i, n}\right)}\right) g\left(U_{T_{i, n}}+d_{T_{i, n}, \xi, U_{T_{i, n}}}, t_{0}-T_{i, n}-\xi\right)\right. \\
& +\mathbf{E}\left(\mathbb{I}_{\left\{\xi \geq \zeta_{j, N_{j}\left(T_{i, n}\right)+1}+T_{j, N_{j}\left(T_{i, n}\right)}-T_{i, n}, \bar{A}_{i, n}\right\}} \Gamma_{j, N_{j}\left(T_{i, n}\right)+1, K} \mid \mathcal{F}_{i, n}\right) \\
& \left.+\mathbf{E}\left(\mathbb{I}_{\left\{\xi \geq \zeta_{i, n+1}, A_{i, n}\right\}} \Gamma_{i, n+1, K} \mid \mathcal{F}_{i, n}\right): \xi \geq 0 \text { is } \mathcal{F}_{i, n} \text {-measurable }\right\} \quad \text { a.s., }
\end{aligned}
$$

where $\bar{F}_{i}=1-F_{i}$ are the survival functions and $A_{i, n}=\left\{\omega: \zeta_{i, n+1}=\zeta_{i, n+1} \wedge\right.$ $\left.\left(\zeta_{j, N_{j}\left(T_{i, n}\right)+1}+T_{j, N_{j}\left(T_{i, n}\right)}-T_{i, n}\right)\right\}$.

(ii) For $n=K, K-1, \ldots, 0, i, j \in\{1,2\}, i \neq j$,

$$
\Gamma_{i, n, K}=\mu_{n} \gamma_{i, K-n}\left(U_{T_{i, n}}, T_{j, N_{j}\left(T_{i, n}\right)}, T_{i, n}\right) \text { a.s., }
$$

where the sequence of the functions $\left\{\gamma_{i, n}(u, s, t), u \in \mathbb{R}, s, t \geq 0, s \leq t\right\}$, using (21), (20) and (22) is defined as follows: $\gamma_{i, 0}(u, s, t)=g\left(u, t_{0}-t\right)$ and

$$
\begin{aligned}
& \gamma_{i, k}(u, s, t)=\sup _{r \geq 0}\left[\bar{F}_{i}(r) \bar{F}_{j}^{t}(t+r-s) g\left(u+c r, t_{0}-t-r\right)\right. \\
& +\int_{0}^{r} d F_{i}(\xi) \int_{\xi+t-s}^{\infty} d F_{j}(\eta) \int_{0}^{u+c \xi} \gamma_{i, k-1}(u+c \xi-x, t+\xi) d H_{i}(x) \\
& \left.+\int_{0}^{r+t-s} d F_{j}(\eta) \int_{\eta+s-t}^{\infty} d F_{i}(\xi) \int_{0}^{u+c(\eta+s-t)} \gamma_{j, k-1}(u+c(\eta+s-t)-x, s+\eta) d H_{j}(x)\right] \\
& \quad j=1,2, \ldots, i=1,2 \text { and } c=c_{1}+c_{2} .
\end{aligned}
$$

Proof. The recursive equation is a direct consequence of the representation theorem for stopping times given by Lemma 1.2 . 
The sequence of functions $\left\{\gamma_{i, n}(u, s, t), u \in \mathbb{R}, s, t \geq 0, s \leq t\right\}$ can be rewritten in simplified form: $\gamma_{i, 0}(u, s, t)=g\left(u, t_{0}-t\right)$ and

$$
\begin{aligned}
& \gamma_{i, k}(u, s, t)=\sup _{r \geq 0}\left[\bar{F}_{i}(r) \bar{F}_{j}^{t}(t+r-s) g\left(u+c r, t_{0}-t-r\right)\right. \\
& +\int_{0}^{r} d F_{i}(\xi) \bar{F}_{j}^{t}(\xi+t-s) \int_{0}^{u+c \xi} \gamma_{i, k-1}(u+c \xi-x, t+\xi) d H_{i}(x) \\
& \left.+\int_{0}^{r+t-s} d F_{j}(\eta) \bar{F}_{i}^{s}(\eta+s-t) \int_{0}^{u+c(\eta+s-t)} \gamma_{j, k-1}(u+c(\eta+s-t)-x, s+\eta) d H_{j}(x)\right] .
\end{aligned}
$$

REMARK. The above equations differ from the ones in Theorem 1 in Muciek (2002) as a result of the different form of the capital assets process $U_{t}$. The optimal value of the problem with the given number of claims $K$ is $\Gamma_{1,0, K}=\Gamma_{2,0, K}$.

The next step is to find the optimal stopping time $\tau_{K}^{*}$. To do this we should analyze the properties of the sequence of the functions $\left\{\gamma_{i, n}, n \geq 0, i \in\{1,2\}\right\}$. Let $B=B[(-\infty,+\infty) \times[0,+\infty) \times[0,+\infty)]$ be the space of all bounded and continuous functions with the norm $\|\delta\|=\sup _{u, s, t}|\delta(u, s, t)|$ and let $B^{0}=\{\delta: \delta(u, s, t)=$ $\delta_{1}(u, s, t) \mathbb{I}_{\left\{s \leq t \leq t_{0}\right\}}$ and $\left.\delta_{1} \in B\right\}$. One should notice that the functions $\left\{\gamma_{i, n}, n \geq 0\right\}$ are included in $B^{0}$. For each $\vec{\delta}=\left(\delta_{1}, \delta_{2}\right) \in B^{0} \times B^{0}$ and any $u \in \mathbb{R}, s, t, r \geq 0$ let

$$
\begin{aligned}
& \left(\vec{\phi}_{\vec{\delta}}(r, u, s, t)\right)_{i}=\bar{F}_{i}(r) \bar{F}_{j}^{t}(t+r-s) g\left(u+c r, t_{0}-t-r\right) \\
& \quad+\int_{0}^{r} d F_{i}(\xi) \bar{F}_{j}^{t}(\xi+t-s) \int_{0}^{u+c \xi} \delta_{i}(u+c \xi-x, t+\xi) d H_{i}(x) \\
& \quad+\int_{0}^{r+t-s} d F_{j}(\eta) \bar{F}_{i}^{s}(\eta+s-t) \int_{0}^{u+c(\eta+s-t)} \delta_{j}(u+c(\eta+s-t)-x, s+\eta) d H_{j}(x) .
\end{aligned}
$$

From the properties of the cumulative distribution function $F .(\cdot)$ it is known that $\vec{\phi}_{\vec{\delta}}(r, u, s, t)$ has at most a countable number of points of discontinuity according to $r$ and is continuous according to $(u, s, t)$ in the case of $g_{1}(\cdot)$ being continuous and $t \neq t_{0}-r$. Therefore, for further considerations we assume that the function $g_{1}(\cdot)$ is bounded and continuous.

Let $\vec{\delta} \in B^{0} \times B^{0}$ and

$$
(\vec{\Phi} \vec{\delta})_{i}(u, s, t)=\sup _{r \geq 0}\left\{\left(\vec{\phi}_{\vec{\delta}}\right)_{i}(r, u, s, t)\right\} \text { for } i \in\{1,2\} \text {. }
$$

Lemma 3.2. For each $\vec{\delta} \in B^{0} \times B^{0}$ we have

$$
(\vec{\Phi} \vec{\delta})_{i}(u, s, t)=\max _{0 \leq r \leq t_{0}-t}\left\{\left(\vec{\phi}_{\vec{\delta}}\right)_{i}(r, u, s, t)\right\} \in B^{0}
$$

and there exists a function $r_{\delta_{i}}(u, s, t)$ such that $(\vec{\Phi} \vec{\delta})_{i}(u, s, t)=\left(\vec{\phi}_{\vec{\delta}}\right)_{i}\left(r_{\delta_{i}}(u, s, t), u, s, t\right)$.

In the subsequent considerations some properties of $\vec{\Phi}$ are presented. For $k=1,2, \ldots$, $i \in\{1,2\}$ and $u \in \mathbb{R}, s, t \geq 0, \gamma_{i, k}(u, s, t)$ can be expressed as follows

$$
\gamma_{i, k}(u, s, t)=\left(\vec{\gamma}_{k}\right)_{i}(u, s, t)= \begin{cases}\left(\vec{\Phi} \vec{\gamma}_{k-1}\right)_{i}(u, s, t) & \text { if } u \geq 0 \text { and } s \leq t \leq t_{0} \\ 0 & \text { otherwise }\end{cases}
$$


From Lemma 3.2 there exist functions $r_{\left(\vec{\gamma}_{k-1}\right)_{i}}(u, s, t)$ such that

$$
\gamma_{i, k}(u, s, t)= \begin{cases}\left(\vec{\phi}_{\vec{\gamma}_{k-1}}\right)_{i}\left(r_{\left(\vec{\gamma}_{k-1}\right)_{i}}(u, s, t), u, s, t\right) & \text { if } u \geq 0 \text { and } s \leq t \leq t_{0}, \\ 0 & \text { otherwise. }\end{cases}
$$

To specify the form of the optimal stopping times $\tau_{i, n, K}^{*}$, we need to define the following random variables $R_{i, s}^{*}=r_{\vec{\gamma}_{K-s+1}}\left(U_{T_{i, s}}, T_{j, N_{j}\left(T_{i, s}\right)}, T_{i, s}\right)$ and $\sigma_{i, n, K}=K \wedge \inf \{s \geq n$ : $\left.R_{i, s}^{\star}<S_{i, s+1}\right\}$. Finally in Corollary 3.3 we present the form of the optimal stopping time.

Corollary 3.3. Let $\tau_{i, n, K}^{*}=T_{i, \sigma_{i, n, K}}+R_{i, \sigma_{i, n, K}}^{\star}$ and $\tau_{K}^{\star}=\tau_{0, K}^{*}=\tau_{i, 0, K}^{*}$, then for all $0 \leq n \leq K$ the following relations hold

$$
\Gamma_{i, n, K}=\mathbf{E}\left(\mathbb{G}\left(\tau_{i, n, K}^{*}\right) \mid \mathcal{F}_{i, n}\right) \text { a.s. } \quad \text { and } \quad \Gamma_{0, K}=\mathbf{E}\left(\mathbb{G}\left(\tau_{i, 0, K}^{*}\right)\right)=\left(\vec{\gamma}_{K}\right)_{i}(a, 0,0) .
$$

This means that $\tau_{i, n, K}^{*}$ and $\tau_{K}^{\star}$ are the optimal stopping times in the classes $\mathcal{T}_{i, n, K}$ and $\mathcal{T}_{0, K}$, respectively.

4. An infinite number of claims. In this section we consider the case of an infinite number of claims and we find the stopping time $\tau^{*}$, which is optimal in the class $\mathcal{T}$. Let us restrict to the cumulative distribution functions such that

Assumption 2. $F_{i}\left(t_{0}\right)<1$ for $i=1,2$.

The following lemma (see Ferenstein and Sierociński (1997)) will play an important role in our considerations.

Lemma 4.1. The operator $\vec{\Phi}: B^{0} \times B^{0} \rightarrow B^{0} \times B^{0}$ defined by 23 is a contraction.

Proof. Let us take $\vec{\delta}^{1}, \vec{\delta}^{2} \in B^{0} \times B^{0}$. By Lemma 3.2 there exists $\vec{\rho}(u, s, t)=r_{\vec{\delta}}(u, s, t)$ such that $(\vec{\Phi} \vec{\delta})_{i}(u, s, t)=\vec{\phi}_{\vec{\delta}_{i}}\left(\vec{\rho}_{i}(u, s, t), u, s, t\right), i=1,2$. Let us notice that $\vec{\phi}_{\vec{\delta}_{i}^{2}}\left(\vec{\rho}_{i}^{2}(u, s, t), u, s, t\right)$ $\geq \vec{\phi}_{\vec{\delta}_{i}^{2}}\left(\vec{\rho}_{i}^{1}(u, s, t), u, s, t\right)$ and it follows that

$$
\begin{aligned}
& \left(\vec{\Phi} \vec{\delta}^{1}\right)_{i}(u, s, t)-\left(\vec{\Phi} \vec{\delta}^{2}\right)_{i}(u, s, t)=\vec{\phi}_{\vec{\delta}_{i}^{1}}\left(\vec{\rho}_{i}^{1}(u, s, t), u, s, t\right)-\vec{\phi}_{\vec{\delta}_{i}^{2}}\left(\vec{\rho}_{i}^{2}(u, s, t), u, s, t\right) \\
& \leq \vec{\phi}_{\vec{\delta}_{i}^{1}}\left(\vec{\rho}_{i}^{1}(u, s, t), u, s, t\right)-\vec{\phi}_{\vec{\delta}_{i}^{2}}\left(\vec{\rho}_{i}^{1}(u, s, t), u, s, t\right) \\
& \leq \int_{0}^{\vec{\rho}_{i}^{1}} d F_{i}(\xi) \bar{F}_{j}^{t}(\xi+t-s) \int_{0}^{u+c \xi}\left[\vec{\delta}_{i}^{1}-\vec{\delta}_{i}^{2}\right](u+c \xi-x, t+\xi) d H_{i}(x) \\
& \quad+\int_{0}^{\vec{\rho}_{i}^{1}+t-s} d F_{j}(\eta) \vec{F}_{i}^{s}(\eta+s-t) \int_{0}^{u+c(\eta+s-t)}\left[\vec{\delta}_{j}^{1}-\vec{\delta}_{j}^{2}\right](u+c(\eta+s-t)-x, s+\eta) d H_{j}(x) \\
& \leq\left\|\vec{\delta}_{i}^{1}-\vec{\delta}_{i}^{2}\right\|\left[\int_{0}^{\vec{\rho}_{i}^{1}} d F_{i}(\xi) \bar{F}_{j}^{t}(\xi+t-s) \int_{0}^{u+c \xi} d H_{i}(x)\right. \\
& \left.\quad+\int_{0}^{\vec{\rho}_{i}^{1}+t-s} d F_{j}(\eta) \bar{F}_{i}^{s}(\eta+s-t) \int_{0}^{u+c(\eta+s-t)} d H_{j}(x)\right] \leq \widetilde{\vartheta}\left\|\vec{\delta}_{i}^{1}-\vec{\delta}_{i}^{2}\right\|,
\end{aligned}
$$


where

$$
\begin{aligned}
\widetilde{\vartheta}= & \sup _{u>0}\left[\int_{0}^{\vec{\rho}_{i}^{1}} d F_{i}(\xi) \bar{F}_{j}^{t}(\xi+t-s) \int_{0}^{u+c \xi} d H_{i}(x)\right. \\
& \left.+\int_{0}^{\vec{\rho}_{i}^{1}+t-s} d F_{j}(\eta) \bar{F}_{i}^{s}(\eta+s-t) \int_{0}^{u+c(\eta+s-t)} d H_{j}(x)\right] \\
\leq & \mathbf{P}\left(S_{1, \cdot} \wedge S_{2, \cdot} \leq t_{0}\right)<1 .
\end{aligned}
$$

Similarly, we get $\left(\vec{\Phi} \vec{\delta}^{2}\right)_{i}(u, s, t)-\left(\vec{\Phi} \vec{\delta}^{1}\right)_{i}(u, s, t) \leq \widetilde{\vartheta}\left\|\delta_{2}-\delta_{1}\right\|$. Hence,

$$
\left\|\left(\vec{\Phi} \vec{\delta}^{1}\right)_{i}(u, s, t)-\left(\vec{\Phi} \vec{\delta}^{2}\right)_{i}(u, s, t)\right\| \leq \widetilde{\vartheta}\left\|\vec{\delta}_{i}^{1}-\vec{\delta}_{i}^{2}\right\|
$$

As $\vec{\gamma}_{0} \in B^{0} \times B^{0}$ we conclude that $\vec{\gamma}_{k} \in B^{0} \times B^{0}$ for all $k$, therefore we can use the Banach fixed point theorem (see Goebel and Kirk (1990), Granas and Dugundji (2003)) and obtain the following lemma

Lemma 4.2. There exists $\vec{\gamma} \in B^{0} \times B^{0}$ such that $\vec{\gamma}=\vec{\Phi} \vec{\gamma}$ and $\lim _{K \rightarrow \infty}\left\|\vec{\gamma}_{K}-\vec{\gamma}\right\|=0$.

Corollary 4.3. $\vec{\gamma}$ is the uniform limit of $\vec{\gamma}_{K}$, when $K$ tends to the infinity.

The considerations of Sections 3 and 4 lead to the following formulation of the optimal strategy after the change of parameters in the risk process.

THEOREM 4.4. If the function $g$ fulfills Assumption 1, $F_{i}$ fulfills Assumption 2 and has the density function $f_{i}, i=1,2$, then

(i) for $n \in \mathbb{N}$ the limit $\tau_{i, n}^{*}=\lim _{K \rightarrow \infty} \tau_{i, n, K}^{*}$ a.s., for $i=1,2$, exists and $\tau_{i, n}^{*}$ is an optimal stopping rule in the set $\mathcal{T} \cap\left\{\tau \geq T_{i, n}\right\}$,

(ii) $E\left(\mathbb{G}\left(\tau_{i, n}^{*}\right) \mid \mathcal{F}_{s, n}\right)=\mu_{i, n} \gamma_{i, n}\left(U\left(T_{i, n}\right), T_{j, N_{j}\left(T_{i, n}\right)}, T_{i, n}\right)$ a.s.

Proof. (i) The stopping rule $\tau_{i, n}^{*}=\lim _{K \rightarrow \infty} \tau_{i, n, K}^{*}$ a.s. exists because $\tau_{i, n, K}^{\star} \leq \tau_{i, n, K+1}^{\star}$.

Let us define the process $\xi(t)=\left(t, U(t), \mathbb{Z}(t), Y_{1}(t), Y_{2}(t), V(t)\right)$, where $Y_{i}(t)=t-$ $T_{i, N_{i}(t)}, \mathbb{Z}(t)=\mathbb{I}_{Z_{N(t)}=1}, V(t)=\mu_{Z(t), N_{Z(t)}(t)}$. By Gikhman and Skorokhod $(2004), \xi(t)$ is a Markov process with the state space $\mathbb{R}^{+} \times \mathbb{R} \times\{0,1\} \times \mathbb{R}^{+} \times \mathbb{R}^{+} \times\{0,1\}$. We express the process $\mathbb{G}(t)$ as $\mathbb{G}(t)=\tilde{g}(\xi(t))$ and calculate the strong generator of $\xi(t)$

$$
\begin{aligned}
(A g)\left(t, u, \nu, y_{1}, y_{2}, v\right)= & v\left\{c g^{\prime}(u)+\nu \frac{f_{1}(y)}{\bar{F}_{1}(y)}\left[\int_{0}^{u} g(u-x) d H_{1}(x)-g(u)\right]\right. \\
& \left.+(1-\nu) \frac{f_{2}(y)}{\bar{F}_{2}(y)}\left[\int_{0}^{u} g(u-x) d H_{2}(x)-g(u)\right]\right\},
\end{aligned}
$$

where $t<t_{0}, y_{i}>0$ for $i=1,2$, and $\nu, v \in\{0,1\}$.

The next step is noting that $g(\xi(t))-g(\xi(0))-\int_{0}^{t}(A g)(\xi(z)) d z$ is a martingale with respect to $\sigma(\xi(z), z \leq t)$, which is the same as $\mathcal{F}_{t}$ (see Davis $(1993)$ ) and applying the optional sampling theorem

$$
E\left\{g\left(\xi\left(\tau_{i, n, K}^{*}\right)\right) \mid \mathcal{F}_{i, n}\right\}-g\left(\xi\left(T_{i, n}\right)\right)=E\left[\int_{T_{i, n}}^{\tau_{i, n, K}^{*}}(A g)(\xi(z)) d z \mid \mathcal{F}_{i, n}\right] \text { a.s. }
$$


It is immediate that

$$
\begin{aligned}
(A g)(\xi(t)) & =\mu_{N(t)}\left\{c g^{\prime}(U(t))\right. \\
& +\mathbb{Z}(t) \frac{f_{1}\left(t-T_{1, N_{1}(t)}\right)}{\bar{F}_{1}\left(t-T_{1, N_{1}(t)}\right)}\left[\int_{0}^{U(t)} g(U(t)-x) d H_{1}(x)-g(U(t))\right] \\
& \left.+(1-\mathbb{Z}(t)) \frac{f_{2}\left(t-T_{2, N_{2}(t)}\right)}{\bar{F}_{2}\left(t-T_{2, N_{2}(t)}\right)}\left[\int_{0}^{U(t)} g(U(t)-x) d H_{2}(x)-g(U(t))\right]\right\},
\end{aligned}
$$

therefore the right side of 24 can be expressed as $E\left\{I_{n, K}^{1} \mid \mathcal{F}_{s, n}\right\}-E\left\{I_{n, K}^{2} \mid \mathcal{F}_{s, n}\right\}$, where

$$
\left.I_{n, K}^{2}=\int_{\widetilde{T}_{n}}^{\widetilde{\tau}_{n, K}^{*}} \mu_{N_{1}(s)} \mu_{N_{2}(z-s)} \frac{f_{2}\left(z-\widetilde{T}_{\left.N_{2}(z-s)\right)}\right.}{\bar{F}_{2}\left(z-\widetilde{T}_{N_{2}(z-s)}\right)} g(U(s, z)) d z\right\} .
$$

We can observe that $I_{n, K}^{1}, I_{n, K}^{2}$ are positive random variables. Let us define a random variable $M=\inf \left\{n \in \mathbb{N}: T_{n}<t_{0}, T_{n+1} \geq t_{0}\right\}$, then

$$
I_{n, K}^{2} \leq \frac{g\left(a+c_{2}\left(t_{0}-s\right)\right)}{\bar{F}_{2}\left(t_{0}\right)} E M \text { a.s., }
$$

where $E M=\sum_{n=0}^{\infty} F_{2}^{*^{(n)}}\left(t_{0}\right) \leq \sum_{n=0}^{\infty}\left[F_{2}\left(t_{0}\right)\right]^{n}<\infty$. By the monotone convergence theorem we see that

$$
E\left[\int_{T_{n}}^{\widetilde{\tau}_{n, K}^{*}}(A \tilde{g})(\xi(s, z)) d z \mid \mathcal{F}_{s, n}\right] \stackrel{K \rightarrow \infty}{\longrightarrow} E\left[\int_{T_{n}}^{\widetilde{\tau}_{n}^{*}}(A \tilde{g})(\xi(s, z)) d z \mid \mathcal{F}_{s, n}\right] \text { a.s. }
$$

Dynkin's formula again yields

$$
E\left[\int_{T_{n}}^{\widetilde{\tau}_{n}^{*}}(A \tilde{g})(\xi(s, z)) d z \mid \mathcal{F}_{s, n}\right]=E\left\{\tilde{g}\left(\xi\left(s, \widetilde{\tau}_{n}^{*}\right)\right) \mid \mathcal{F}_{s, n}\right\}-\tilde{g}\left(\xi\left(s, T_{n}\right)\right) \text { a.s. }
$$

Combining these with 24, , 25) we obtain

$$
E\left\{\tilde{g}\left(\xi\left(s, \widetilde{\tau}_{n, K}^{*}\right)\right) \mid \mathcal{F}_{s, n}\right\} \stackrel{K \rightarrow \infty}{\longrightarrow} E\left\{\tilde{g}\left(\xi\left(s, \widetilde{\tau}_{n}^{*}\right)\right) \mid \mathcal{F}_{s, n}\right\} \text { a.s. }
$$

The task is now to prove that $\widetilde{\tau}_{n}^{*}$ is optimal in the $\mathcal{T}^{s} \cap\left\{\tau: \tau \geq T_{n}\right\}$. Let $\tau$ be any stopping rule $\tau \in \mathcal{T}^{s} \cap\left\{\tau: \tau \geq T_{n}\right\}$, then from optimality of $\widetilde{\tau}_{n, K}^{*}$ we get

$$
E\left\{\tilde{g}\left(\xi\left(s, \widetilde{\tau}_{n, K}^{*}\right)\right) \mid \mathcal{F}_{s, n}\right\} \geq E\left\{\tilde{g}\left(\xi\left(s, \tau \wedge T_{K}\right)\right) \mid \mathcal{F}_{s, n}\right\} \text { a.s. }
$$

In the same manner like before, we can see that $E\left\{\tilde{g}\left(\xi\left(s, \widetilde{\tau}_{n}^{*}\right)\right) \mid \mathcal{F}_{s, n}\right\} \geq E\left\{\tilde{g}(\xi(s, \tau)) \mid \mathcal{F}_{s, n}\right\}$, which completes the proof.

(ii) Applying Theorem 3.1 and $(19)$ we deduce that

$$
E\left\{\tilde{g}\left(\xi\left(s, \widetilde{\tau}_{n, K}^{*}\right)\right) \mid \mathcal{F}_{s, n}\right\}=E\left\{\mathbb{G}\left(s, \widetilde{\tau}_{n, K}^{*}\right) \mid \mathcal{F}_{s, n}\right\}=\mu_{N_{1}(s)} \mu_{s, n} \widetilde{\gamma}_{K-n}\left(U\left(s, T_{n}\right), T_{n}\right) \text { a.s. }
$$

Combining Lemma 4.1 and (27) we obtain

$$
E\left\{\tilde{g}\left(\xi\left(s, \widetilde{\tau}_{n, K}^{*}\right)\right) \mid \mathcal{F}_{s, n}\right\} \stackrel{K \rightarrow \infty}{\longrightarrow} E\left\{\tilde{g}\left(\xi\left(s, \widetilde{\tau}^{*}\right)\right) \mid \mathcal{F}_{s, n}\right\}=\mu_{N_{1}(s)} \mu_{s, n} \widetilde{\gamma}\left(U\left(s, T_{n}\right), T_{n}\right) \text { a.s. }
$$

To end this section we notice that the optimal stopping time for the second stop is equal to $\widetilde{\tau}^{*}=\widetilde{\tau}_{0}^{*} \in \mathcal{T}^{s}$, where $\widetilde{\tau}_{0}^{*}=\lim _{K \rightarrow \infty} \widetilde{\tau}_{0, K}^{*}$ and the conditional value function of the optimal stopping problem after moment $s$ is given by

$$
J(s)=E\left\{\mathbb{G}\left(s, \widetilde{\tau}_{0}^{*}\right) \mid \mathcal{F}_{s, 0}\right\}=\mu_{N_{1}(s)} \mu_{s, 0} \widetilde{\gamma}\left(U\left(s, T_{0}\right), T_{0}\right)=\mu_{N_{1}(s)} \widetilde{\gamma}\left(U_{1}(s), s\right) \quad \text { a.s. }
$$


5. Final remarks. The topic of the paper can be extended in many ways, mainly by fitting the risk process to the economic environment and the possible decision in the insurance practice. An example of related research is Azcue and Muler (2005). Recently, Ferenstein and Pasternak-Winiarski (2010) have taken into account the possibility of abrupt changes in the risk process modeling only one insurance contract or aggregated assets.

Acknowledgements. I have benefited from discussions with Dr. Anna Karpowicz on an early version of the manuscript, for which I am grateful.

\section{References}

P. Azcue and N. Muler (2005), Optimal reinsurance and dividend distribution policies in the Cramér-Lundberg model, Math. Finance 15 (2), 261-308.

F. Boshuizen and J. Gouweleeuw (1993), General optimal stopping theorems for semi-Markov processes, Adv. in Appl. Probab. 4, 825-846.

F. A. Boshuizen (1994), A general framework for optimal stopping problems associated with multivariate point processes, and applications, Sequential Anal. 13 (4), 351-365.

P. Brémaud (1981), Point Processes and Queues, Martingale Dynamics, Springer, New York.

M. H. A. Davis (1993), Markov Models and Optimization, Chapman and Hall, New York.

E. B. Dynkin (1969), Game variant of a problem on optimal stopping, Soviet Math. Doklady 10, $270-274$.

R. Eidukjavicjus (1979), Optimalnaia ostanovka markovskoi cepi dvumia momentami ostanovki, Lit. Mat. Sb. 13, 181-183.

E. Z. Ferenstein and A. Pasternak-Winiarski (2010), Optimal stopping of a risk process with disruption and interest rates, In: Breton, M., Szajowski, K. (eds.), Advances in Dynamic Games: Differential and Stochastic Games: Theory, Application and Numerical Methods, Annals of the International Society of Dynamic Games 11, Birkhäuser, Boston.

E. Z. Ferenstein and A. Sierociński (1997), Optimal stopping of a risk process, Applicationes Mathematicae 24 (3), 335-342.

I. Gikhman and A. Skorokhod (2004), The Theory of Stochastic Processes, II, Springer, Berlin.

K. Goebel and W. Kirk (1990), Topics in Metric Fixed Point Theory, Cambridge Studies in Advanced Mathematics 28, Cambridge University Press, Cambridge.

A. Granas and J. Dugundji (2003), Fixed Point Theory, Springer Monographs in Mathematics, Springer, New York, NY.

G. Haggstrom (1967), Optimal sequential procedures when more than one stop is required, Ann. Math. Stat. 38, 1618-1626.

M. Jacobsen (2006), Point Process Theory and Applications. Marked Point and Piecewise Deterministic Processes, Probability and Its Applications 7, Birkhäuser, Boston.

A. Karpowicz and K. Szajowski (2007), Double optimal stopping of a risk process, Stochastics: An International Journal of Probability and Stochastics Processes 79 (1-2), 155-167.

B. Muciek (2002), Optimal stopping of a risk process: Model with interest rates, J. Appl. Prob. $39,261-270$

B. Muciek and K. Szajowski (2007), Optimal stopping of a risk process when claims are covered immediately, In: Mathematical Economics, RIMS Kôkyûroku 1557, 132-139.

M. Nikolaev (1979), Obobshchennyie posledovatelnyie procedury, Litov. Mat. Sb. 191, 35-44. 
M. Nikolaev (1998), Optimal multi-stopping rules, Obozr. Prikl. Prom. Mat. 5 (2), 309-348.

T. Rolski, H. Schmidli, V. Schmidt and J. Teugels (1998), Stochastic Processes for Insurance and Finance, John Wiley \& Sons, Chichester.

A. Schöttl (1998), Optimal stopping of a risk reserve process with interest and cost rates, J. Appl. Prob. 35, 115-123.

W. Stadje (1985), On multiple stopping rules, Optimization 16, 401-418. 
